\title{
GESTIÓN DEL CONOCIMIENTO Y GERENCIA DE PROYECTOS EN LA INVESTIGACIÓN UNIVERSITARIA: CASO DE ESTUDIO EN EL DEPARTAMENTO TECNOLOGÍA DE SERVICIOS DE LA UNIVERSIDAD SIMÓN BOLÍVAR DE VENEZUELA
}

KNOWLEDGE MANAGEMENT AND PROJECTS MANAGEMENT IN THE UNIVERSITY RESEARCH: STUDY CASE IN THE TECHNOLOGY OF SERVICES DEPARTMENT OF THE SIMON BOLIVAR UNIVERSITY OF VENEZUELA

María Daniela Gómez Suárez mdgomez@usb.ve 
Para citar este artículo:

Gómez Suárez, M. D. (2020). Gestión del conocimiento y gerencia de proyectos en la investigación universitaria: Caso de estudio en el departamento tecnología de servicios de la Universidad Simón Bolívar de Venezuela. ESPACIO I+D, INNOVACIÓN MÁS DESARROLLO, 9(24). https://doi.org/10.31644/IMASD.24.2020.a02

\section{RESUMEN}

Con los proyectos realizados en cualquier organización y los procesos para llevarlos a cabo, que consideran la gestión de la integración, del alcance, del tiempo, de los costos, de la calidad, de los recursos humanos, de las comunicaciones, de los riesgos, de las adquisiciones y de los interesados, es posible generar nuevo conocimiento organizacional y aprovechar el que ya existe. En el caso particular de las universidades, donde se realizan constantemente proyectos de diversa índole, con resultados que se extienden más allá de estas instituciones, de forma que impactan a la sociedad en general, el utilizar herramientas y metodologías apropiadas para la gestión de proyectos es fundamental ya que contribuye a mantener el rigor científico necesario, más aún cuando los procesos vinculados se pueden considerar como factores fundamentales para la gestión del conocimiento en las dimensiones de: personas, procesos, contenidos y tecnologías de la información y comunicación. De este modo, se realizó una investigación de tipo descriptivo con un diseño no experimental, que tuvo como objetivo general determinar la gestión del conocimiento realizado por los profesores del Departamento Tecnología de Servicios de la Universidad Simón Bolívar Sede Litoral, del Estado La Guaira en Venezuela, para lo cual fueron considerados los procesos y áreas de conocimiento para la gerencia de los proyectos de investigación que realizan, y se diagnosticó por medio de una autoevaluación cómo los profesores gerencian sus proyectos para luego relacionar la información obtenida con los factores que promueven la gestión del conocimiento.

\section{Palabras Clave}

Gestión del conocimiento, gestión de proyectos, procesos, áreas de conocimiento, proyectos universitarios. 
With the projects realized in any organization and the processes to carry them out, that consider the management of the integration, scope, time, cost, quality, human resources, communications, risk, procurement and stakeholder, it is possible to generate new organizational knowledge and take advantage of that already exists. In the particular case of universities, where different kinds of projects are constantly realized, with results that extend beyond these institutions in a way that impacts society in general, using appropriate tools and methodologies for project management is essential as it contributes to maintaining the necessary scientific rigor, even more so when the linked processes can be considered as fundamental factors for knowledge management in the dimensions of: people, processes, content and information and communication technologies. Thus and so, a descriptive research was realized with a non-experimental design, that had as general objective to determine the knowledge management carried out by the professors of the Technology of Services Department of the Simon Bolivar University Litoral Campus, of the State of La Guaira in Venezuela, for which the processes and areas of knowledge were considered for the management of the research projects done it, and it was diagnosed through a self-evaluation how professors managed their projects to then relate the information obtained to the factors that promote knowledge management.

\section{Keywords}

Knowledge management, project management, processes, knowledge areas, university projects. 
$\mathrm{E}$ 1 proceso de investigación universitaria se sujeta al rigor científico y metodológico, lo que coincide con la definición de Cobo (2006) sobre la gestión del conocimiento, por medio del cual el conocimiento organizacional, tras generarse, se captura, transforma y utiliza; además, se debe considerar que a la hora de investigar se realizan tanto actividades rutinarias como proyectos o programas temporales, con los cuales es posible dar respuesta a las inquietudes personales y profesionales de los investigadores, de la institución a la que pertenecen o de la sociedad en general.

Por lo tanto, en función del rigor científico, además de seguir los procedimientos pertinentes para cada área de estudio, cuando de proyectos se trata, hay que utilizar las herramientas y metodologías propias de la gerencia de proyectos que "abarca niveles estratégicos, tácticos y operativos como responsables directos de su adecuada gestión” (Álvarez, 2016: 348) sin olvidar que la gestión del conocimiento tiene, como señala Cobo (2006), una perspectiva práctica y operativa que se debe considerar.

Pero en algunas ocasiones, la formación de base de los profesores incide en que, a la hora de investigar, no se sigan las metodologías adecuadas o se consideren las herramientas acordes para gestionar proyectos, sobre todo si éstas abarcan áreas de conocimiento que superan su formación profesional, por ejemplo, no realizar una buena gestión del tiempo por no usar cronogramas o descuidar los costos por no realizar un presupuesto. Adicionalmente, existen factores que Figueredo (2017) denomina como tropiezos del investigador universitario que son: factor tiempo, ausencia de una definición clara de carrera dentro de la institución, escaso presupuesto, aspecto anímico y la falta de coincidencia entre los intereses investigativos personales y de la institución, los cuales impactan también en las investigaciones realizadas y que, en algunos casos y cada vez con mayor frecuencia, se potencian dada la actual situación política, económica y social venezolana, que repercute en las universidades y en todo su personal.

Dadas estas condiciones, se hace indispensable que los proyectos investigativos que aún se realizan en las universidades del país, puedan satisfacer las necesidades que los motivaron, además de mantener el rigor científico y metodológico requerido, que sean gestionados adecuadamente al tener en cuenta los procesos que éstos requieren para llegar a término dentro de las restricciones existentes, que se encuentran acotadas por las áreas de conocimiento vinculadas a la gerencia de proyectos; de ahí se desprende el objetivo general de esta investigación, que es del tipo descriptivo con un diseño no experimental, donde por medio de un caso de estudio, se pretende determinar la gestión del conocimiento realizado por los profesores del Departamento Tecnología de Servicios de la Universidad Simón Bolívar, Sede Litoral del Estado La Guaira de Venezuela, para ello fueron tomados en cuenta los procesos y áreas de conocimiento para la 
gerencia de los proyectos de investigación que realizan y se diagnosticó, por medio de una autoevaluación, cómo los profesores gerencian sus proyectos, para posteriormente relacionar la información obtenida con los factores que promueven la gestión del conocimiento.

\section{GESTIÓN DEL CONOCIMIENTO}

En el caso particular de los profesores, estos son "agentes sociales con mayor responsabilidad en la materialización hacia lo interno y visibilización hacia lo externo del cientificismo y de la función investigación, atribuida a las universidades" (Figueredo, 2017: 325), para así dar cumplimiento con el compromiso social de estas instituciones, lo cual deben hacer bajo un enfoque organizacional y estratégico que se explica a continuación. Para Cegarra y Martínez (2017), una estrategia es un instrumento para la acción y supone el acto de dirigir o gestionar con la finalidad de cumplir unas metas, ajustadas a la incertidumbre del mundo organizacional y del entorno altamente cambiante; por otra parte, nadie puede dudar actualmente que los activos intangibles son elementos estratégicos en las organizaciones que contribuyen con su crecimiento, siendo cada vez más relevante su gestión, especialmente en el caso de los países en desarrollo porque "ha pasado a ser una herramienta para la mejora del desempeño" (González y Rodríguez, 2016: 12), y como afirman Quintas et al (1997) es un proceso de gestión continua de todo tipo de conocimiento, con el cual es posible satisfacer necesidades existentes y emergentes, que identifica y utiliza activos de conocimiento presentes con el fin de desarrollar nuevas oportunidades.

La estrategia empresarial, en su enfoque más clásico, considera los recursos y capacidades propios de la organización, a diferencia de las estrategias basadas en el conocimiento que, como indica Sveiby (1997; citado por Cegarra y Martínez, 2017), se sustentan y tienen como ventaja: un alto nivel de personalización, que el conocimiento se enfoca como procedimiento, que hay beneficios derivados de la eficacia y la inversión realizada en la formación del personal. Por su parte González y Rodríguez (2016: 19), indican que la gestión del conocimiento considera "actividades tales como: iniciar, generar, utilizar, modelizar, transferir y retroalimentar dicho conocimiento para los fines propios y los objetivos estratégicos", lo cual es requerido para que el conocimiento se convierta en patrimonio organizativo, para ello primero se debe conjugar el conocimiento individual dadas las competencias personales, estudios, destrezas y repetición de hechos, el cual debe ser compartido y distribuido de forma tácita con otros miembros de la organización para crear así su propia memoria interna (Cegarra y Martínez, 2017), sin dejar de lado que todos los procesos relacionados con esta gestión, además de generar, buscar, almacenar y transferir el conocimiento, buscan 
aumentar la productividad y la competitividad (García, 2002; citado por González y Rodríguez, 2016).

Olivares (2002; citado por Machado et al, 2007) aporta que en algunas ocasiones no existe una gestión coordinada entre las funciones de la universidad, ya que algunos docentes carecen de la formación gerencial necesaria, lo que se complica aún más sin una gestión del conocimiento que permita afrontar desafíos propios de la organización y del entorno brindando "herramientas y procesos que les permitan generar nuevo conocimiento y rentabilizar el conocimiento existente, mejorando así el rendimiento de los trabajadores y, por tanto, de la propia organización" (Rodríguez-Gómez y Gairín, 2015: 73). La gestión del conocimiento, según Angulo (2016), debe incluir un flujo permanente y administrado de conocimientos dentro de la organización, ya que esto estimula la innovación, mejora los procesos de toma de decisiones y genera nuevos conocimientos.

Optimizar la gestión del conocimiento implica un aprendizaje organizativo que permita operacionalizar la "generación y aprovechamiento permanente del conocimiento existente y desarrollado" (Rodríguez-Gómez y Gairín, 2015: 76), lo que debe motivar a un proceso de mejora continua y, más aún en contextos formativos como el de las universidades, que sobrepase el campo individual de los investigadores y abarque a toda la institución. Para lograr todo esto, la gestión del conocimiento debe sustentarse en cuatro pilares que influyen en su eficacia: personas, procesos, contenidos y tecnologías de la información y comunicación (Gómez- Vargas y García, 2015); a continuación, en la tabla 1 se presentan los componentes fundamentales de la gestión del conocimiento. 
Tabla 1

Componentes de la gestión del conocimiento

\begin{tabular}{cc}
\hline $\begin{array}{c}\text { Componentes de la gestión } \\
\text { del conocimiento }\end{array}$ & Descripción de los componentes de la gestión del conocimiento \\
\hline Personas & $\begin{array}{c}\text { Recursos humanos } \\
\text { Cultura organizativa interiorizada }\end{array}$ \\
\hline Procesos & $\begin{array}{c}\text { Contexto en el que se produce la gestión del conocimiento } \\
\text { Visión, misión de la organización. Estrategias de negocio }\end{array}$ \\
Metodologías y rutinas organizativas que llevan a cabo las personas \\
Conocimiento asociado a cada área
\end{tabular}

Los componentes de la gestión del conocimiento siguen una ruta que se inicia con las personas al adquirir y utilizar el conocimiento desde etapas iniciales de aprendizaje hasta la mejora continua, creando procesos y contenidos que, bajo los medios y ambiente propicios, sean almacenados, distribuidos y captados por los miembros de la organización, convirtiéndolo en patrimonio de esta.

Como indican Gómez-Vargas y García (2015), los estudios realizados sobre gestión del conocimiento en centros de educación superior y específicamente en el ámbito de la investigación científica, se enfocan en analizar solo una o algunas variables de los procesos de la gestión del conocimiento, particularmente aquellos relacionados con la creación de éste y las formas de transferirlo, pero en cualquier tipo de organización y a través de los componentes de la gestión del conocimiento, se pueden desarrollar y analizar capacidades propias y, en el caso de las universidades, esto puede hacerse con los proyectos de investigación que realizan, específicamente evaluando las fortalezas y debilidades de los profesores en materia de gerencia de proyectos, por lo cual, es lógico considerar métricas de esta temática a la hora de evaluar la gestión del conocimiento. 


\section{GERENCIA DE PROYECTOS}

La gerencia de proyectos es parte de un proceso más amplio que la organización, como un todo, debe analizar y adaptar a las estrategias que se desean desarrollar (Álvarez, 2016). Es necesario acotar que, como señalan Barbosa y Moura (2013), los proyectos permiten obtener resultados que van más allá del ámbito de gestión de la rutina diaria, por lo tanto no deben dejarse al azar, especialmente por sus particularidades, las cuales se resumen en que: sus resultados son de características únicas, sujetas a una serie de restricciones operativas (con jerarquización de actividades interdependientes) y de recursos (humanos, materiales y de tiempo), sus equipos son multidisciplinarios, la ejecución se ve afectada por la incertidumbre y los riesgos asociados a cada actividad, dada la interacción con distintas variables organizacionales y del entorno.

El Project Management Institute define a la gerencia de proyectos como "la aplicación de conocimientos, habilidades, herramientas y técnicas a las actividades del proyecto para cumplir con los requisitos del mismo" (2013: 5); para eso, deben realizarse una serie de procesos que se encuentran vinculados con las áreas de conocimiento de la gestión de proyectos. En el caso de los proyectos de investigación realizados desde las universidades, el impacto que éstos poseen abarca más allá de los intereses particulares de los investigadores y de la institución, ya que son extensivos hacia toda la sociedad, además de convertirse, como afirman Gutiérrez-Vallejo et al (2016), "en un punto de encuentro para que los miembros de la comunidad educativa alcancen acuerdos de trabajo mancomunado y consciente del poder que tienen cuando actúan en equipo y de manera organizada".

Los grupos de procesos de la gerencia de proyectos, según el Project Management Institute (2013) son:

- Iniciación: incluye todas las actividades o tareas que hay que realizar para convertir una idea en un proyecto viable (Ollé y Cerezuela, 2017), además de definir y autorizar el proyecto o una fase del mismo.

- Planificación: abarca la definición del alcance del proyecto, así como "la elaboración de unos objetivos alcanzables, basados en los recursos disponibles y en los condicionamientos económicos, sociales y culturales" (Pérez, 2016: 37), así como el curso de acciones necesarias para alcanzarlos de forma realista.

- Ejecución: es la integración de recursos para llevar a cabo el plan de gestión a fin de satisfacer las especificaciones de este, completando el entregable del proyecto (Wilson, 2015), al realizar todo el trabajo que fue previamente definido. 
- Monitoreo y control: considera aquellas actividades relacionadas a la supervisión y regulación del avance regular del progreso del proyecto, a fin de identificar variaciones respecto del plan de gestión e iniciar los cambios o acciones correctivas (Wallace, 2014: 22), de hacer falta, en el momento indicado.

- $\quad$ Cierre: procesos que implican la finalización de todas las actividades del proyecto así como la formalización de la aceptación del producto, servicio o resultado, para lo cual el gerente encargado del proyecto debe asegurarse que desde el punto de vista técnico y legal, el proyecto ha terminado (Turner, 2016).

Las áreas de conocimiento de la gerencia de proyectos resumen las herramientas y técnicas necesarias para obtener resultados en cada grupo de procesos, además de "definir las variables que intervienen en un proyecto y que inciden en el producto, servicio o resultado esperado" (Ugas, 2008: 78); para el Project Management Institute (2013) son diez estas áreas: gestión de la integración, gestión del alcance, gestión del tiempo, gestión de los costos, gestión de la calidad, gestión de recursos humanos, gestión de las comunicaciones, gestión de los riesgos, gestión de las adquisiciones y gestión de los interesados; cada una de ellas requiere, por parte de los involucrados en los proyectos, competencias relacionadas con conocimientos, habilidades y destrezas personales. Es así, que:

- Gestión de la integración: "incluye los procesos y actividades necesarios para identificar, definir, combinar, unificar y coordinar" las distintas áreas de conocimiento (Project Management Institute, 2013: 4), para obtener el resultado esperado.

- Gestión del alcance: definición del trabajo y de los requerimientos fundamentales del proyecto (Torres y Torres, 2014), con el fin de garantizar que se incluya todo el trabajo necesario para lograr el resultado tal como fue planificado.

- Gestión del tiempo: considera los procesos necesarios para culminar las actividades dentro del plazo estipulado, al definir, secuenciar, estimar recursos y duración de actividades, y desarrollar el cronograma (Másmela, 2014).

- Gestión de los costos: consiste en elaborar el presupuesto y en "hacer que el costo real quede dentro de los límites" aprobados (Zandhuis et al, 2014: 93), además incluye los procesos vinculados a la obtención del financiamiento.

- Gestión de la calidad: para verificar que el proyecto satisfaga las necesidades para las cuales fue planificado, cumpliendo fundamentalmente con lo que Toala et al (2019) denominan requisitos del cliente. 
- Gestión de los recursos humanos: implica la asignación de roles y responsabilidades que permitan concretar los objetivos, así como su participación a la hora de planificar y tomar decisiones del proyecto (Romano y Yacuzzi, 2011).

- Gestión de las comunicaciones: garantiza que la información requerida por todos los involucrados en un proyecto sea recopilada, almacenada y distribuida de forma oportuna y por los medios adecuados, para que haya comodidad y confianza durante todo el proyecto (Turner, 2016).

- Gestión de los riesgos: se realiza para identificar, analizar, planificar, anticiparse y controlar las respuestas ante posibles situaciones riesgosas, todo esto basado en ideas conceptuales sobre cómo serán los entregables del proyecto (Wilson, 2015).

- Gestión de las adquisiciones: incluye el alquiler o contratación de todos aquellos bienes y servicios requeridos por el equipo de trabajo y que sean "necesarios para que el proyecto evolucione" (Estrada, 2015: 86).

- Gestión de los interesados: permite identificar a las "personas, grupos u organizaciones que pueden afectar o ser afectados por el proyecto", para así analizar sus expectativas y desarrollar estrategias basadas en su posible impacto (Project Management Institute, 2013: 391).

A continuación, en la figura 1 se presenta un resumen de las áreas de conocimiento y como se vinculan con los procesos antes mencionados. 


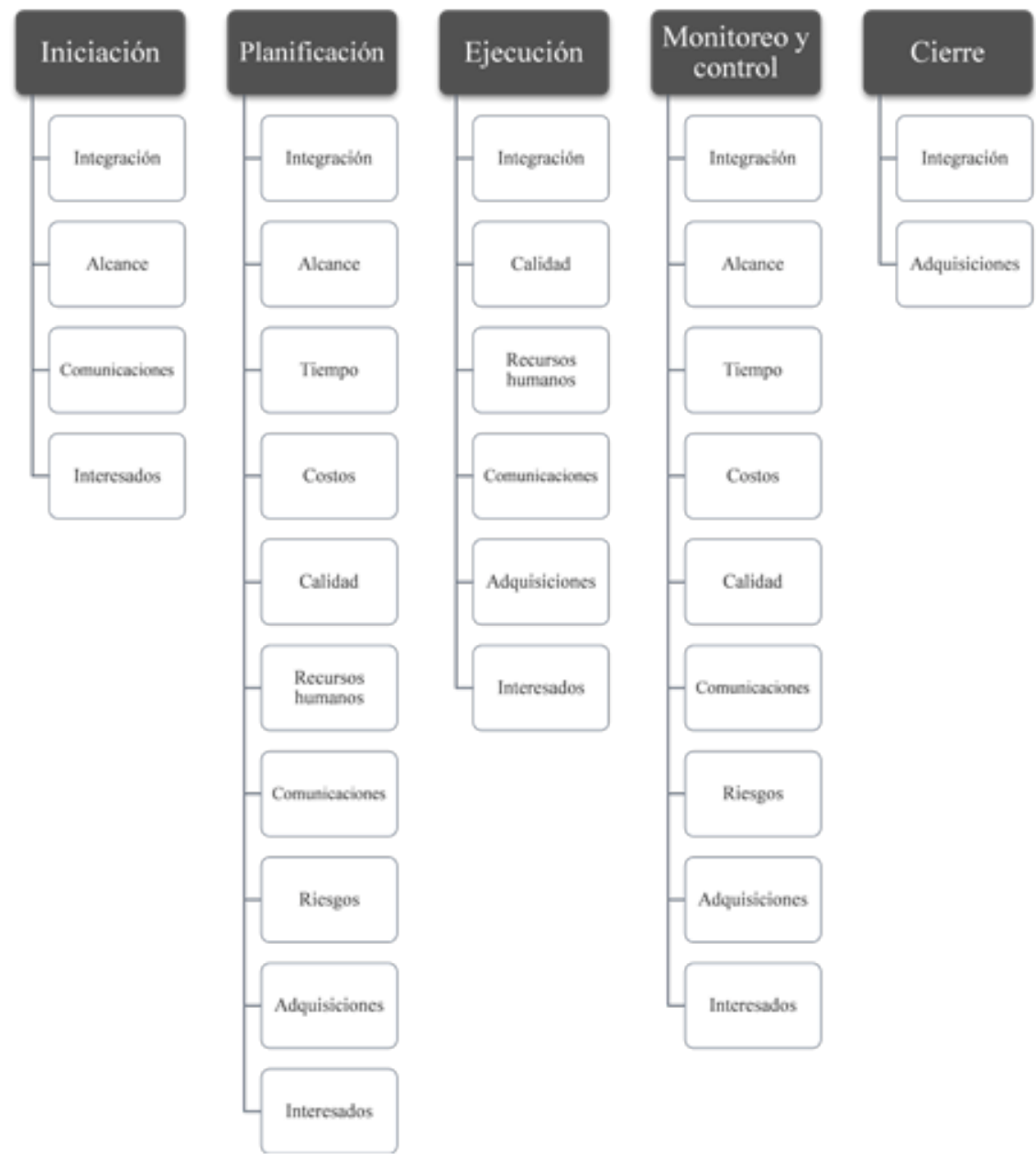

Figura 1. Vinculación entre las áreas de conocimiento y los procesos de gestión de proyectos. Fuente: adaptado de Mariné y Rodríguez, 2014 (citados por Ollé y Cerezuela, 2017) y del

Project Management Institute, 2013

\section{METODOLOGÍA}

La presente investigación es de tipo descriptivo con un diseño no experimental, ya que se pretendió describir, registrar, analizar e interpretar "la naturaleza actual, composición o procesos de los fenómenos” (Rodríguez, 2005: 24), lo cual se logró con el objetivo general de determinar la gestión del conocimiento realizado por los profesores del Departamento Tecnología de Servicios de la Universidad Simón Bolívar Sede Litoral, al considerar los procesos y áreas de conocimiento para la gerencia de los proyectos de investigación que realizan. Para alcanzar el objetivo general antes mencionado se consideran los siguientes objetivos específicos: 
- Diagnosticar por medio de una autoevaluación cómo los profesores gerencian sus proyectos.

- Relacionar la información obtenida con los factores que promueven la gestión del conocimiento.

La población en estudio son los profesores del referido departamento universitario, mientras que la muestra se recogió de forma intencional para así seleccionar solo a los profesores que cumplan con los criterios teóricos y no estadísticos que permitan responder las preguntas de investigación (Fàbregues et al, 2016), siendo así que la muestra quedó conformada por aquellos profesores que por su tipo de contratación realizan proyectos de investigación dentro de la universidad (ya que hay algunos que solo se dedican a la docencia dentro de unas horas estipuladas de clases).

El instrumento de recolección de datos, con el cual se obtuvo la información relacionada con los proyectos realizados por los profesores, es un cuestionario de autoevaluación propuesto por Palacios (2009), en el cual, para cada área de conocimiento y cada proceso de la gestión de proyectos, propone una serie de preguntas, las cuales se evalúan considerando una ponderación por escalas. Posteriormente, la información recogida permitió relacionar los factores que promueven la gestión del conocimiento, a través de los proyectos realizados por los profesores.

\section{CASO DE ESTUDIO}

Los profesores del Departamento Tecnología de Servicios de la Universidad Simón Bolívar que, dado su tipo de contratación, participaron en el estudio para autoevaluar la gestión de proyectos de investigación realizados, se agrupan en lo que se denominan "secciones" vinculadas a sus carreras de formación en pregrado y postgrado, a los cursos de profesionalización que han realizado y a las materias que dictan dentro de la institución. Como se puede apreciar en la tabla 2, algunos de los profesores tienen bases metodológicas o herramientas que les pueden permitir gestionar proyectos en general, sin necesariamente tener estudios en el campo, dado el carácter multidisciplinario de las áreas de conocimiento que requieren los proyectos. 


\section{Tabla 2}

Secciones del Departamento Tecnología de Servicios y conocimientos de los profesores

\begin{tabular}{|c|c|c|}
\hline Secciones & $\begin{array}{l}\text { Porcentaje de profesores del } \\
\text { Departamento por Sección }\end{array}$ & $\begin{array}{c}\text { Temáticas de Pregrado, Postgrado } \\
\text { y cursos de formación }\end{array}$ \\
\hline $\begin{array}{c}\text { Sección de Servicios Turísticos y } \\
\text { Hoteleros }\end{array}$ & $24 \%$ & $\begin{array}{c}\text { Turismo } \\
\text { Gestión de la hospitalidad } \\
\text { Química } \\
\text { Nutrición } \\
\text { Gastronomía } \\
\text { Patrimonio cultural y turístico } \\
\text { Mercadeo } \\
\text { Economía } \\
\text { Administración } \\
\text { Derecho }\end{array}$ \\
\hline $\begin{array}{c}\text { Sección de Servicios Aduaneros y } \\
\text { Comerciales }\end{array}$ & $33 \%$ & $\begin{array}{c}\text { Economía } \\
\text { Ciencias fiscales } \\
\text { Administración aduanera } \\
\text { Relaciones internacionales } \\
\text { Mercadeo internacional } \\
\text { Negocios internacionales } \\
\text { Gerencia de proyectos } \\
\text { Derecho }\end{array}$ \\
\hline $\begin{array}{l}\text { Sección de Tecnologías Empre- } \\
\text { sariales y del Transporte }\end{array}$ & $43 \%$ & $\begin{array}{c}\text { Administración } \\
\text { Contabilidad } \\
\text { Economía } \\
\text { Administración del transporte } \\
\text { Recursos humanos } \\
\text { Finanzas } \\
\text { Gerencia general } \\
\text { Gerencia de proyectos Ingeniería } \\
\text { industrial } \\
\text { Ingeniería de sistemas } \\
\text { Computación } \\
\text { Derecho } \\
\text { Coaching }\end{array}$ \\
\hline
\end{tabular}

Fuente: elaboración propia, 2019

Como se aprecia en la tabla antes presentada, hay profesores que manifiestan tener conocimientos en gerencia de proyectos, pero hay muchos otros que aunque no se hayan formado específicamente en el tema, sus estudios les brindan los conocimientos necesarios para al menos gestionar una o varias de las áreas de conocimiento de la gerencia de proyectos, lo que se corresponde con lo informado en el gráfico 1 que muestra la percepción de los profesores acerca de los conocimientos previos en gerencia de proyectos que poseen. 


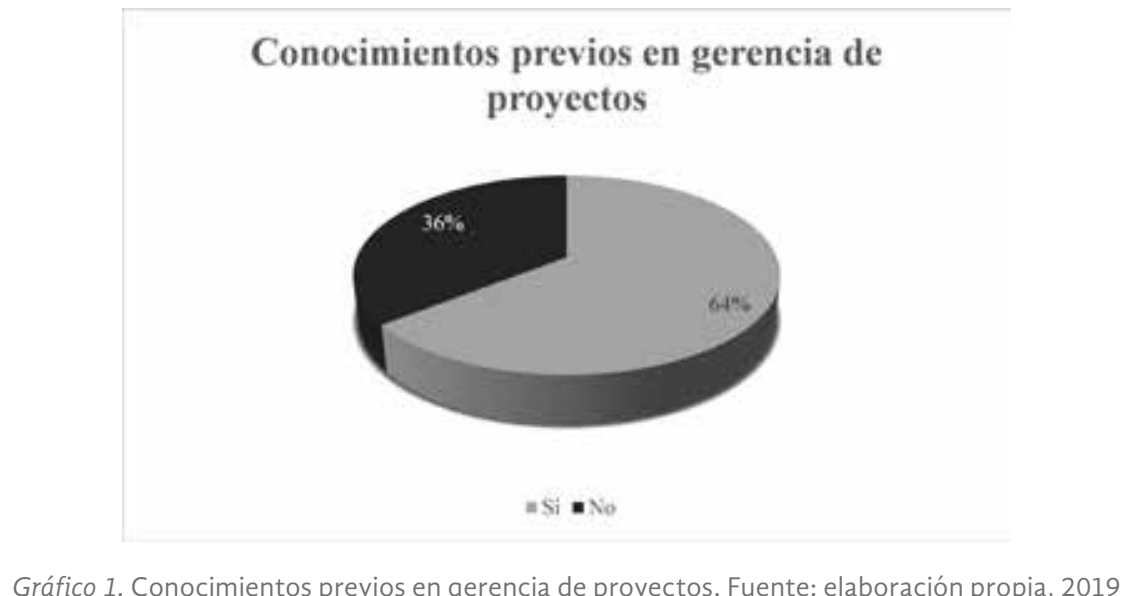

Para determinar la gestión del conocimiento, considerando indicadores de la gerencia de proyectos, primero debió realizarse la autoevaluación por parte de los profesores acerca de los procesos que llevan a cabo y las áreas de conocimiento vinculadas con los proyectos, lo cual fue realizado siguiendo las preguntas del cuestionario propuesto por Palacios (2009), aclarando que para la época de realización del mismo, aún el Project Management Institute no había considerado a la gestión de los involucrados como décima área de conocimiento, sin embargo, el cuestionario realizado incluyó preguntas vinculadas a procesos que consideran a los involucrados, por lo que es pertinente para esta evaluación. Posteriormente, se relacionaron los indicadores emanados de la autoevaluación con los componentes fundamentales de la gestión del conocimiento ("personas", "procesos", "contenidos" y "tecnologías de información y comunicación”), para así determinar cómo los profesores realizan la gestión del conocimiento a través de los proyectos de investigación que realizan.

A continuación, en la tabla 3 se observa como el componente "personas" presenta debilidades en todos los procesos vinculados con el recurso humano, lo que implica que se deben mejorar la planificación de los roles necesarios para cumplir con las tareas y la asignación de los responsables de cada actividad, así como el desarrollo y motivación del equipo de trabajo y la evaluación de su desempeño; esas debilidades se mantienen cuando el profesor se evalúa como gerente de los proyectos que realiza, al no ponderar bien el trabajo que realiza como integrador de un plan que abarque todas las áreas de conocimiento. De estos resultados hay que destacar que: la mayoría de los consultados indicó como respuesta "no aplica" para sus casos particulares y es congruente con la política de la universidad que exige a los profesores noveles y que no hayan ingresado aún al escalafón universitario, a trabajar en sus proyectos de investigación de forma individual y, considerando que la mayoría de los profesores del departamento se encuentran en esa condición, no hay personal o equipo de trabajo con el que cuenten a la hora 
de investigar en etapas iniciales de su carrera universitaria, además llama la atención que, a pesar de haber profesionales expertos en gerencia y recursos humanos, existan debilidades significativas en esas áreas.

\section{Tabla 3}

Componente "personas" de la gestión del conocimiento vinculado al cuestionario de autoevaluación de gerencia de proyectos

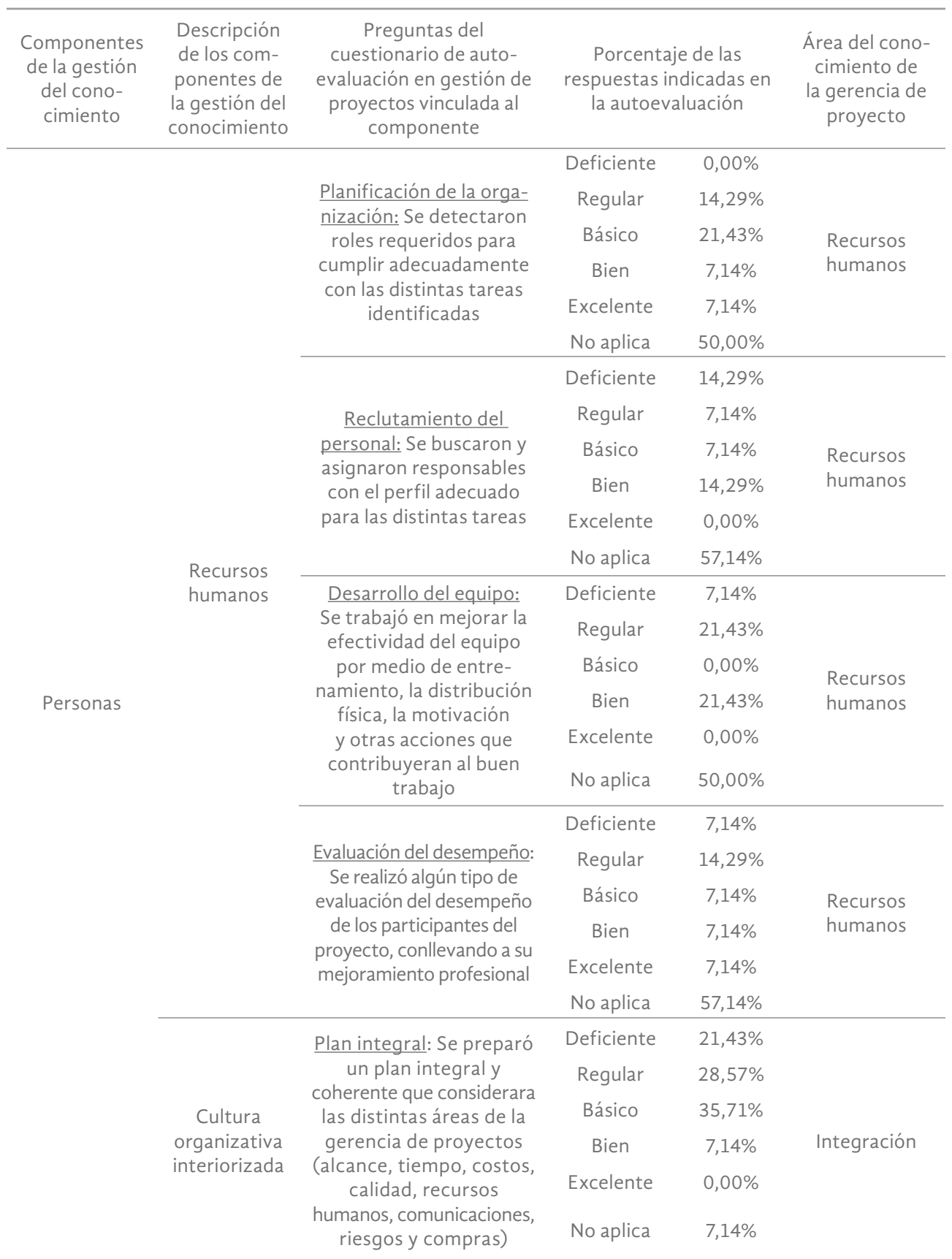

Fuente: elaboración propia, 2019 
Al autoevaluar el componente "procesos" (ver tabla 4), los resultados son similares al del componente anterior, ya que a pesar de contar con profesores expertos en temas relacionados con las áreas de conocimiento: alcance, tiempo, costos, calidad, riesgos e integración de proyectos, se presentan carencias que afectan el contexto en el que se produce la gestión del conocimiento, al subestimar la probabilidad de ocurrencia de riesgos, así como el impacto y el diseño de un plan de respuestas ante los mismos; también, la planificación estratégica de la organización se ve afectada, ya que como gerentes de los proyectos que realizan, no se considera un plan global que agrupe la ejecución de todas las actividades en todos los procesos y áreas. Lo mismo ocurre cuando se consideran las metodologías y rutinas organizativas llevadas a cabo, ya que se evidenció que no existe un sistema para manejar los cambios correcta y oportunamente; los costos no siempre han sido controlados (esto posiblemente por el contexto hiperinflacionario del país que los desvirtúa y hace que los presupuestos queden desactualizados en horas, incluso si estos se realizan en divisas), no se consideraron indicadores ni acciones correctivas dados sus resultados para mantener los requerimientos de los proyectos; lo mismo ocurrió en el caso de los riesgos ante los cuales no hubo un control certero de respuestas, ni para los cambios que afectaban de forma integral a los proyectos. Mención especial en este punto, lo tienen la verificación del alcance para monitorear que las actividades contempladas en el plan se estuviesen cumpliendo, así como la definición de éstas, que de forma global tuvieron deficiencias, aunque casi la mitad de los profesores manifestó realizar una gestión autocalificada como "bien" por ellos.

La situación no es distinta cuando se evalúan los conocimientos asociados a cada área, ya que la mayoría de las veces no se realizaron estudios de factibilidad económica y social de los proyectos, ni se construyó y controló un cronograma claro en cuanto a fechas de realización de actividades, ni se aplicaron metodologías para llevar a cabo acciones correctivas en cuanto a cambios en los cronogramas; en cuanto a los costos, no se creó un presupuesto coherente adaptado al cronograma ni hubo, en muchos casos, un manejo adecuado de las entradas y salidas de dinero generadas por los proyectos; además, faltaron especificaciones claras de la calidad que se ven reflejadas en la falta de planificación y ausencia de indicadores que midieran los resultados finales, según las especificaciones proyectadas. 
Tabla 4

Componente "procesos" de la gestión del conocimiento vinculado al cuestionario de autoevaluación de gerencia de proyectos

\begin{tabular}{|c|c|c|c|c|c|}
\hline \multirow[t]{2}{*}{$\begin{array}{l}\text { Componentes } \\
\text { de la gestión } \\
\text { del cono- } \\
\text { cimiento }\end{array}$} & \multirow[t]{2}{*}{$\begin{array}{l}\text { Descripción de } \\
\text { los compo- } \\
\text { nentes de la } \\
\text { gestión del } \\
\text { conocimiento }\end{array}$} & \multirow[t]{2}{*}{$\begin{array}{l}\text { Preguntas del cues- } \\
\text { tionario de auto- } \\
\text { evaluación en gestión } \\
\text { de proyectos vinculada } \\
\text { al componente }\end{array}$} & \multicolumn{2}{|c|}{$\begin{array}{c}\text { Porcentaje de las } \\
\text { respuestas indicadas en la } \\
\text { autoevaluación }\end{array}$} & \multirow[t]{2}{*}{$\begin{array}{l}\text { Área del cono- } \\
\text { cimiento de } \\
\text { la gerencia de } \\
\text { proyecto }\end{array}$} \\
\hline & & & Deficiente & $35,71 \%$ & \\
\hline & \multirow{11}{*}{$\begin{array}{l}\text { Contexto en el } \\
\text { que se produce } \\
\text { la gestión del } \\
\text { conocimiento }\end{array}$} & \multirow{5}{*}{$\begin{array}{l}\text { Calificación: Se evaluó } \\
\text { la probabilidad de que } \\
\text { ocurrieran los eventos } \\
\text { riesgosos detectados y } \\
\text { su impacto o efecto }\end{array}$} & Regular & $28,57 \%$ & \multirow{5}{*}{ Riesgos } \\
\hline & & & Básico & $0,00 \%$ & \\
\hline & & & Bien & $7,14 \%$ & \\
\hline & & & Excelente & $0,00 \%$ & \\
\hline & & & No aplica & $28,57 \%$ & \\
\hline & & \multirow{6}{*}{$\begin{array}{l}\text { Plan de respuesta: Se } \\
\text { diseñaron planes de } \\
\text { respuesta para adelan- } \\
\text { tarse a los riesgos, ya sea } \\
\text { para mitigar o absorber } \\
\text { el riesgo }\end{array}$} & Deficiente & $35,71 \%$ & \multirow{6}{*}{ Riesgos } \\
\hline & & & Regular & $21,43 \%$ & \\
\hline & & & Básico & $7,14 \%$ & \\
\hline & & & Bien & $7,14 \%$ & \\
\hline & & & Excelente & $0,00 \%$ & \\
\hline & & & No aplica & $28,57 \%$ & \\
\hline & \multirow{6}{*}{$\begin{array}{l}\text { Visión y } \\
\text { misión de la } \\
\text { organización. } \\
\text { Estrategias de } \\
\text { negocio }\end{array}$} & \multirow{6}{*}{$\begin{array}{l}\text { Ejecución global: } \\
\text { Se consideraron los } \\
\text { principales elementos } \\
\text { del plan en la ejecución } \\
\text { de las actividades y se } \\
\text { manejaron integrada- } \\
\text { mente como un todo }\end{array}$} & Deficiente & $28,57 \%$ & \multirow{6}{*}{ Integración } \\
\hline & & & Regular & $28,57 \%$ & \\
\hline & & & Básico & $28,57 \%$ & \\
\hline & & & Bien & $7,14 \%$ & \\
\hline & & & Excelente & $0,00 \%$ & \\
\hline \multirow{19}{*}{ Procesos } & & & No aplica & $7,14 \%$ & \\
\hline & \multirow{18}{*}{$\begin{array}{l}\text { Metodología y } \\
\text { rutinas orga- } \\
\text { nizativas que } \\
\text { llevan a cabo } \\
\text { las personas }\end{array}$} & \multirow{6}{*}{$\begin{array}{l}\text { Verificación del } \\
\text { alcance: Se chequeó a } \\
\text { medida que se ejecu- } \\
\text { taba el proyecto que } \\
\text { se estaban haciendo } \\
\text { las actividades con- } \\
\text { templadas }\end{array}$} & Deficiente & $7,14 \%$ & \multirow{6}{*}{ Alcance } \\
\hline & & & Regular & $14,29 \%$ & \\
\hline & & & Básico & $28,57 \%$ & \\
\hline & & & Bien & $42,86 \%$ & \\
\hline & & & Excelente & $7,14 \%$ & \\
\hline & & & No aplica & $0,00 \%$ & \\
\hline & & & Deficiente & $14,29 \%$ & \\
\hline & & Control del alcance: Se & Regular & $50,00 \%$ & \\
\hline & & manejar los cambios de & Básico & $14,29 \%$ & I \\
\hline & & alcance correctamente, & Bien & $14,29 \%$ & \\
\hline & & correctivas & Excelente & $7,14 \%$ & \\
\hline & & & No aplica & $0,00 \%$ & \\
\hline & & & Deficiente & $14,29 \%$ & \\
\hline & & Definición de activi- & Regular & $7,14 \%$ & \\
\hline & & dades: Se delimitaron & Básico & $28,57 \%$ & \\
\hline & & que derivaron productos & Bien & $42,86 \%$ & тетро \\
\hline & & específicos & Excelente & $7,14 \%$ & \\
\hline & & & No aplica & $0,00 \%$ & \\
\hline
\end{tabular}


rutinas orga-

nizativas que

llevan a cabo

las personas

Procesos

\begin{tabular}{|c|c|c|c|}
\hline \multirow{6}{*}{$\begin{array}{l}\text { Control de costos: Se } \\
\text { controló el presupuesto } \\
\text { tomando las acciones } \\
\text { correctivas cuando } \\
\text { surgieron cambios en el } \\
\text { presupuesto }\end{array}$} & Deficiente & $28,57 \%$ & \multirow{6}{*}{ Costos } \\
\hline & Regular & $21,43 \%$ & \\
\hline & Básico & $7,14 \%$ & \\
\hline & Bien & $14,29 \%$ & \\
\hline & Excelente & $7,14 \%$ & \\
\hline & No aplica & $21,43 \%$ & \\
\hline \multirow{6}{*}{$\begin{array}{l}\text { Control de calidad: Se } \\
\text { midieron indicadores } \\
\text { y se tomaron acciones } \\
\text { correctivas cuando se } \\
\text { detectaron desviaciones }\end{array}$} & Deficiente & $35,71 \%$ & \multirow{6}{*}{ Calidad } \\
\hline & Regular & $14,29 \%$ & \\
\hline & Básico & $21,43 \%$ & \\
\hline & Bien & $21,43 \%$ & \\
\hline & Excelente & $0,00 \%$ & \\
\hline & No aplica & $7,14 \%$ & \\
\hline \multirow{6}{*}{$\begin{array}{l}\text { Control de respuestas: } \\
\text { Se hicieron revisiones } \\
\text { periódicas de riesgos, } \\
\text { verificando posibles } \\
\text { riesgos y activando } \\
\text { contingencias }\end{array}$} & Deficiente & $42,86 \%$ & \multirow{6}{*}{ Riesgos } \\
\hline & Regular & $14,29 \%$ & \\
\hline & Básico & $7,14 \%$ & \\
\hline & Bien & $7,14 \%$ & \\
\hline & Excelente & $0,00 \%$ & \\
\hline & No aplica & $28,57 \%$ & \\
\hline \multirow{6}{*}{$\begin{array}{l}\text { Control global: Se } \\
\text { manejaron integral- } \\
\text { mente los cambios y sus } \\
\text { efectos sobre cada área } \\
\text { del proyecto, revisán- } \\
\text { dose las variaciones y } \\
\text { sus repercusiones }\end{array}$} & Deficiente & $28,57 \%$ & \multirow{6}{*}{ Integración } \\
\hline & Regular & $21,43 \%$ & \\
\hline & Básico & $35,71 \%$ & \\
\hline & Bien & $7,14 \%$ & \\
\hline & Excelente & $0,00 \%$ & \\
\hline & No aplica & $7,14 \%$ & \\
\hline \multirow{6}{*}{$\begin{array}{l}\text { Iniciación: Se formuló y } \\
\text { evaluó económicamente } \\
\text { el proyecto o se deter- } \\
\text { minó su impacto social } \\
\text { para decidir su ejecución, } \\
\text { con una descripción del } \\
\text { proyecto indicando su } \\
\text { relevancia y los } \\
\text { productos deseados }\end{array}$} & Deficiente & $42,86 \%$ & \multirow{6}{*}{ Alcance } \\
\hline & Regular & $14,29 \%$ & \\
\hline & Básico & $42,86 \%$ & \\
\hline & Bien & $0,00 \%$ & \\
\hline & Excelente & $0,00 \%$ & \\
\hline & No aplica & $0,00 \%$ & \\
\hline \multirow{6}{*}{$\begin{array}{l}\text { Programación de activi- } \\
\text { dades: Se construyó un } \\
\text { cronograma coherente } \\
\text { que permitiera ver } \\
\text { cuando se harían las } \\
\text { actividades }\end{array}$} & Deficiente & $7,14 \%$ & \multirow{6}{*}{ Tiempo } \\
\hline & Regular & $14,29 \%$ & \\
\hline & Básico & $35,71 \%$ & \\
\hline & Bien & $35,71 \%$ & \\
\hline & Excelente & $7,14 \%$ & \\
\hline & No aplica & $0,00 \%$ & \\
\hline \multirow{6}{*}{$\begin{array}{l}\text { Control de crono- } \\
\text { grama: Se aplicó alguna } \\
\text { metodología para medir } \\
\text { desviaciones del } \\
\text { avance del trabajo y sus } \\
\text { posibles correcciones }\end{array}$} & Deficiente & $28,57 \%$ & \multirow{6}{*}{ Tiempo } \\
\hline & Regular & $28,57 \%$ & \\
\hline & Básico & $28,57 \%$ & \\
\hline & Bien & $7,14 \%$ & \\
\hline & Excelente & $0,00 \%$ & \\
\hline & No aplica & $7,14 \%$ & \\
\hline
\end{tabular}




\begin{tabular}{|c|c|c|c|c|c|}
\hline \multirow{18}{*}{ Procesos } & \multirow{18}{*}{$\begin{array}{c}\text { Conocimiento } \\
\text { asociado a } \\
\text { cada área }\end{array}$} & \multirow{6}{*}{$\begin{array}{l}\text { Presupuesto: Se creó } \\
\text { un presupuesto coher- } \\
\text { ente que permitiera } \\
\text { ajustar los distintos } \\
\text { costos estimados a las } \\
\text { fechas programadas }\end{array}$} & Deficiente & $21,43 \%$ & \multirow{6}{*}{ Costos } \\
\hline & & & Regular & $14,29 \%$ & \\
\hline & & & Básico & $28,57 \%$ & \\
\hline & & & Bien & $14,29 \%$ & \\
\hline & & & Excelente & $0,00 \%$ & \\
\hline & & & No aplica & $21,43 \%$ & \\
\hline & & \multirow{6}{*}{$\begin{array}{l}\text { Manejo de la tesorería: } \\
\text { Se manejó adecuada- } \\
\text { mente las entradas y } \\
\text { salidas de dinero en el } \\
\text { proyecto }\end{array}$} & Deficiente & $35,71 \%$ & \multirow{6}{*}{ Costos } \\
\hline & & & Regular & $14,29 \%$ & \\
\hline & & & Básico & $0,00 \%$ & \\
\hline & & & Bien & $21,43 \%$ & \\
\hline & & & Excelente & $7,14 \%$ & \\
\hline & & & No aplica & $21,43 \%$ & \\
\hline & & \multirow{6}{*}{$\begin{array}{l}\text { Planificación de la } \\
\text { calidad: Se especifi- } \\
\text { caron los resultados } \\
\text { que deben ofrecer los } \\
\text { productos finales con } \\
\text { indicadores de gestión }\end{array}$} & Deficiente & $28,57 \%$ & \multirow{6}{*}{ Calidad } \\
\hline & & & Regular & $14,29 \%$ & \\
\hline & & & Básico & $14,29 \%$ & \\
\hline & & & Bien & $28,57 \%$ & \\
\hline & & & Excelente & $0,00 \%$ & \\
\hline & & & No aplica & $14,29 \%$ & \\
\hline
\end{tabular}

Fuente: elaboración propia, 2019

Como se aprecia en la tabla 5, a la hora de autoevaluar el componente "contenidos", los resultados mejoran en algunos aspectos en comparación con componentes previos, particularmente si se habla de información recopilada, por ejemplo, en planes y definición del alcance ante los cuales se usaron metodologías que permitieron identificar a las actividades y a los involucrados, así como secuenciar y determinar prelaciones de actividades por realizar. El resto de la autoevaluación fue consistente con resultados previos, ya que las fallas se mantienen a la hora de estimar duración de actividades en conjunto con los involucrados en ellas, planificar recursos y estimar sus costos, así como para la identificación de los riesgos asociados a las mismas y de las necesidades que deben ser cubiertas por compras que deben ser plasmadas en un plan de requerimientos, así como la elección de los proveedores más adecuados. Si se consideran los lenguajes documentales controlados y que son soportados a través de formatos específicos como el plan de compras con los contratos requeridos y el cierre de estos, tampoco el resultado de la autoevaluación considera una efectiva gestión de esos procesos. 
Tabla 5

Componente "contenidos" de la gestión del conocimiento vinculado al cuestionario de autoevaluación de gerencia de proyectos

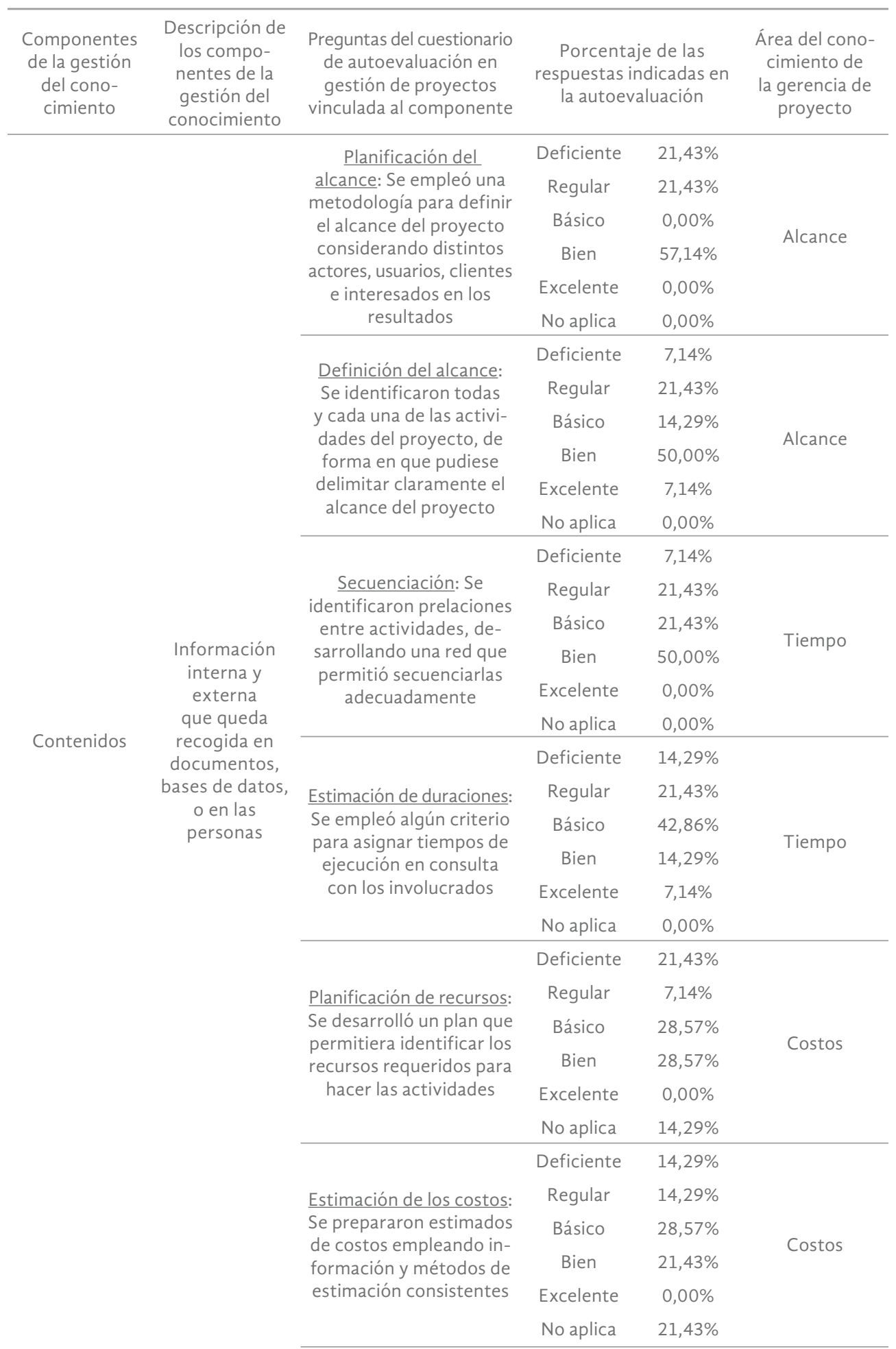




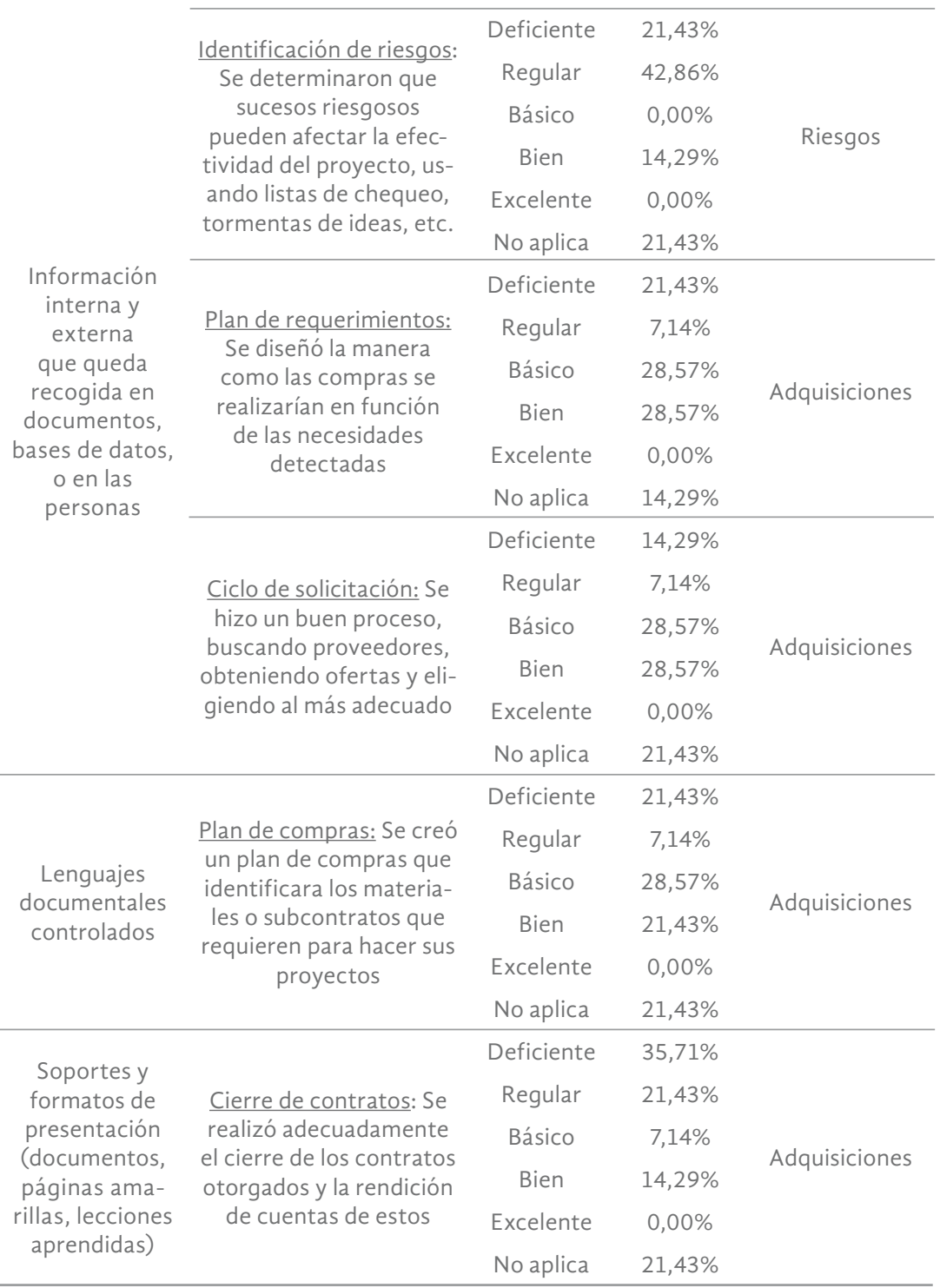

Fuente: elaboración propia, 2019

Los medios evaluados para recoger, almacenar y distribuir los datos, información y conocimiento (presentados en la tabla 6), muestran que los procesos autoevaluados con las mejores ponderaciones se corresponden con la gestión de las comunicaciones, particularmente a la hora de planificarla, identificando las necesidades de información y cuándo debieron hacerse reportes periódicos del progreso de los proyectos para los involucrados. Ya el resto de los procesos vinculados a este componente, tuvieron respuestas concentradas entre los resultados deficientes, regulares y básicos, afectando el aseguramiento de la calidad y cumplimiento de especificaciones, el seguimiento de los contratos y de las comunicaciones, pero esta vez en los procesos relacionados con la 
distribución oportuna y óptima de la información, así como con la recolección de las lecciones aprendidas al cierre de los proyectos.

\section{Tabla 6}

Componente "tecnologías de información y comunicación" de la gestión del conocimiento vinculado al cuestionario de autoevaluación de gerencia de proyectos

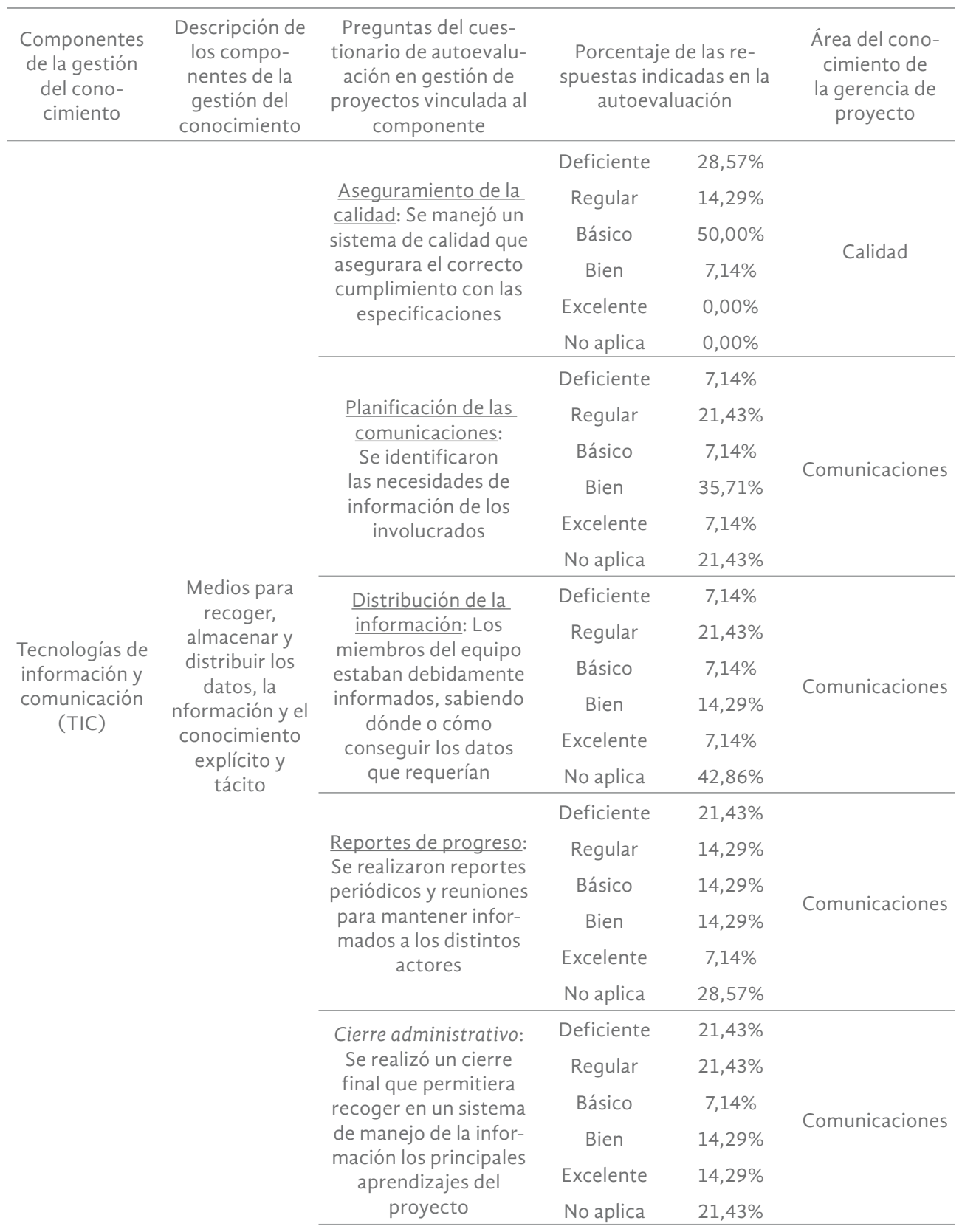




\begin{tabular}{|c|c|c|c|c|c|}
\hline \multirow{6}{*}{$\begin{array}{l}\text { Tecnologías de } \\
\text { información y } \\
\text { comunicación } \\
\text { (TIC) }\end{array}$} & \multirow{6}{*}{$\begin{array}{c}\text { Alineación con } \\
\text { estrategia y } \\
\text { necesidades de } \\
\text { la organización, } \\
\text { especialmente } \\
\text { las requeridas } \\
\text { al estudiar los } \\
\text { otros tres com- } \\
\text { ponentes }\end{array}$} & \multirow{6}{*}{$\begin{array}{l}\text { Administración de } \\
\text { contratos: Se efectuó } \\
\text { una labor eficiente en } \\
\text { el manejo e inspección } \\
\text { de los contratos, con } \\
\text { algún sistema para } \\
\text { hacerle seguimiento }\end{array}$} & Deficiente & $21,43 \%$ & \multirow{6}{*}{ Adquisiciones } \\
\hline & & & Regular & $21,43 \%$ & \\
\hline & & & Básico & $28,57 \%$ & \\
\hline & & & Bien & $0,00 \%$ & \\
\hline & & & Excelente & $0,00 \%$ & \\
\hline & & & No aplica & $28,57 \%$ & \\
\hline
\end{tabular}

Fuente: elaboración propia, 2019

\section{CONCLUSIÓN}

Cada vez se reconoce más la importancia que tiene, para cualquier organización, considerar como factores clave de éxito los procesos y componentes que conducen hacia una óptima gestión del conocimiento, porque es un elemento estratégico organizacional, más aún en las universidades, que tienen como fin último la creación y difusión de conocimiento, y el impacto de las actividades y proyectos realizados tienen influencia a niveles local, nacional e incluso internacional. Sin embargo, si en una organización no se ha concientizado la necesidad e importancia de esto, sea por problemas coyunturales, como por ejemplo la situación del país que prioriza otro tipo de gestiones, que dan peso a las acciones urgentes sobre las planificadas, o si se suman los denominados tropiezos del investigador, se termina afectando uno de los pilares de la carrera profesoral dentro de las universidades; lo anterior incide en que los procesos no sean optimizados, por lo tanto, los objetivos no se alcanzan y los resultados obtenidos no tienen ni el impacto ni el alcance deseados.

Para poder corregir y mejorar la gestión realizada, sea de proyectos o de conocimientos, es necesario identificar primero lo que se está haciendo y cómo se está haciendo, para así detectar fallas, que permitan luego tomar las acciones correctivas que sean necesarias. Como se apreció en el caso de estudio, las fallas en la gestión de proyectos pudieron vincularse con la gestión del conocimiento, al evaluar el impacto que tienen actualmente en la institución algunos factores facilitadores e inhibidores sobre la gestión, los cuales están vinculados con las acciones y procesos de proyectos realizados por los profesores y a nivel institucional, la información que manejan y el formato en que la recopilan y comparten por medio de las tecnologías de la información y comunicación.

Si se analiza el componente "personas", es notorio que actualmente el profesorado que participa en los proyectos de investigación del departamento analizado, no gestiona adecuadamente los procesos relacionados con los recursos humanos de los proyectos, descuidando hasta ahora la asignación de roles y responsabilidades, el desarrollo de equipos de 
trabajo y la retroalimentación necesaria, lo que también se observa con la gestión de la integración a la hora de considerarse cada profesor como gerente de los proyectos que realiza. La situación es similar cuando se autoevalúa el componente "procesos", ya que las debilidades manifestadas en el estudio, para generar conocimiento, se vinculan con la gestión de las áreas de gestión de proyectos de alcance, tiempo, costos, calidad, riesgos e integración, siendo así que para los profesores del departamento hay problemas para verificar el alcance, carecen de sistemas o metodologías de gestión de cambios así como de indicadores de gestión, no verifican el cumplimiento cabal del plan, las actividades, cronogramas, costos y presupuestos, dejan de lado acciones correctivas diseñadas para mantener los requerimientos del proyecto, no evaluaron la factibilidad económica y social, subestimaron los riesgos a la hora de identificarlos y de diseñar un plan de respuesta ante ellos, y como gerentes de proyectos no hubo una visión global que vinculara a la planificación estratégica organizacional con todos los procesos y áreas de gestión de proyectos, ni se valoró el impacto de los cambios dentro del proyecto como un todo.

En el caso del componente "contenidos", los profesores manifestaron planificar los proyectos y definir el alcance como medio para recopilar información, también indicaron que utilizan metodologías para identificar, secuenciar y priorizar actividades e involucrados; las fallas se presentan cuando tienen que estimar la duración de actividades, planificar recursos, estimar costos, identificar riesgos, planificar compras que abarquen todas las necesidades, elegir proveedores y gestionar contratos.

Cuando se considera el componente "tecnologías de información y comunicación”, hay una mejor autoevaluación en los procesos que permiten identificar necesidades de información y reportar periódicamente el progreso a los involucrados, sin embargo hay resultados que comprometen el aseguramiento de la calidad y el cumplimiento de especificaciones (y por lo tanto el resultado final de los proyectos); tampoco realizan seguimiento de contratos, ni la distribución oportuna y optima de la información para que todos los involucrados estén notificados de los avances y resultados, y tampoco recolectan las lecciones aprendidas al finalizar los proyectos, por lo que no existe un cuerpo de conocimientos a disposición de la comunidad universitaria.

Es necesario aclarar que en ningún momento la presente investigación tiene como propósito desvirtuar el trabajo realizado por los profesores del departamento, al cual precisamente pertenece la investigadora, además se debe puntualizar que el sistema de control exigido por el Decanato y las Coordinaciones de investigación por áreas de estudio de la Universidad Simón Bolívar, requiere que se entreguen resultados para justificar el dinero invertido, así como para renovar los contratos de los profesores, por lo que 
se verificó que los proyectos en su gran mayoría cumplieron los objetivos para los cuales fueron propuestos; con este trabajo se evidencia que: a) de forma intuitiva muchos profesores realizaron los procesos requeridos y b) existen necesidades de formación en gerencia de proyectos, por lo que es ideal que dentro de las actuales restricciones presupuestarias, la universidad apoye a los profesores brindando la capacitación necesaria y fomentando una cultura organizacional con procesos dinámicos y multidisciplinarios, soportados en una óptima gestión conformando un cuerpo de conocimientos común que abarque a toda la comunidad universitaria y que refuerce la motivación de los profesores por distintos medios para que estos alcancen un reconocimiento profesional, como académicos y con resultados de calidad que impacten de forma positiva a toda la sociedad. Es necesario aclarar, que esta situación puede ser similar en otros contextos universitarios, por lo que se insta a realizar una investigación similar en el resto de la institución y en otras universidades. 


\section{REFERENCIAS CONSULTADAS}

Álvarez, M. (2016). El poder de la gestión de proyectos en la cultura organizacional. Revista Ciencias Estratégicas. Vol. 24, N 36, pág. 345-363. República de Colombia.

Angulo, R. (2016). Gestión del conocimiento y aprendizaje organizacional: una visión integral. Revista Informes Psicológicos. Vol. 17, $\mathrm{N}^{\circ}$ 1, pág. 5370. República de Colombia.

Barbosa, E. y Moura, D. (2013). Proyectos educativos y sociales. Planificación, gestión, seguimiento y evaluación. Reino de España: Narcea Ediciones.

Cegarra, J. y Martínez, A. (2017). Gestión del conocimiento una ventaja competitiva. Reino de España: ESIC Editorial.

Cobo, A. (2006), Modelo de capital intelectual de la Consejería de Educación de la Junta de Andalucía. Indicadores de capital humano y gestión del conocimiento. Tesis Doctoral, Universidad de Málaga, Reino de España, [Tesis en línea] Disponible en: http://www.biblioteca.uma.es/bbldoc/ tesisuma/16754621.pdf

Estrada, J. (2015). Análisis de la gestión de proyectos a nivel mundial. Palermo Business Review. 12, 61-98. República Argentina.

Fàbregues, S., Meneses, J., Rodríguez-Gómez, D. y Paré, M. (2016). Técnicas de investigación social y educativa. Reino de España: Editorial uoc.

Figueredo, C. (2017). La gerencia avanzada en el proceso de investigación científica universitaria. Revista Scientific. Vol. 2, Nº 6, pág. 322-336. República del Ecuador.

Gómez-Vargas, M. y García, M. (2015). Factores influyentes de la gestión del conocimiento en el contexto de la investigación universitaria. Revista Información, cultura y sociedad. $N^{\circ} 33$, pág. 29-46. República Argentina.

González, J. y Rodríguez, M. (2016). Gestión del conocimiento, capital intelectual e indicadores aplicados. Reino de España: Ediciones Díaz de Santos.

Gutiérrez-Vallejo, E. Gracia-Chancay, J. y Quiñonez-Mosquera, J. (2016). Liderazgo y gerencia de proyectos educativos y sociales. Revista Dominio de las Ciencias. Vol. 2, $\mathrm{N}^{\circ}$ 4, pág. 274-283. República del Ecuador.

Machado, F., Reyes, L. y Rietveldt, F. y Luquez, P. (2007). Articulación de las funciones universitarias: un marco de transformación académica. Revista Formación Gerencial, año 6, $\mathrm{N}^{\circ}$ 6, pág. 64-87. República Bolivariana de Venezuela.

Másmela, R. (2014). Como implementar sistemas para la gestión de proyectos en organizaciones para el desarrollo de software guiados por un modelo de mejora continua. República de Colombia: Grafiweb.

Ollé, C.; Cerezuela, B. (2017). Gestión de proyectos paso a paso. Reino de España: Editorial Uoc. 
Palacios, L. (2009). Principios esenciales para realizar proyectos. Un enfoque latino. 4ta. edición. Caracas, República Bolivariana de Venezuela: Universidad Católica Andrés Bello.

Pérez, G. (2016). Diseño de proyectos sociales: aplicaciones prácticas para su planificación, gestión y evaluación. Reino de España: Narcea Ediciones.

Project Management Institute (2013). Guía de los fundamentos para la dirección de proyectos: Guía del Рмвок. Quinta edición. Estados Unidos de América: PMi Publications.

Quintas, P., Lefrere, P. y Jones, G. (1997). Knowledge Management: a strategic agenda. Revista Long Range Planning. Vol. 30, N 3, pág. 385-391. Reino de los Países Bajos.

Rodríguez, E. (2005). Metodología de la investigación. La creatividad, el rigor del estudio y la integridad son factores que transforman a un estudiante en un profesionista de éxito. Estados Unidos Mexicanos: Universidad Juárez Autónoma de Tabasco.

Rodríguez -Gómez, D. y Gairín, J. (2015). Innovación, aprendizaje organizativo y gestión del conocimiento en las instituciones educativas. Revista Educación. Vol. 24, N 46, pág. 73-90. República del Perú.

Romano, G. y Yacuzzi, E. (2011). Elementos de la gestión de proyectos. Serie documentos de trabajo de la Universidad del cema. 449, 1-32. República Argentina.

Toala, M., Romero, R., Ganchozo, M., Álvarez, C., Jaime, M., Pinargote, J. Romero, V. y Bazurto, J. (2019). Introducción a la gestión de proyectos. Reino de España: Editorial Área de Innovación y Desarrollo.

Torres, Z. y Torres, H. (2014). Administración de proyectos. Estados Unidos Mexicanos: Grupo Editorial Patria.

Turner, R. (2016). Gower Handbook of Project Management. Quinta edición. Estados Unidos de América: Editorial Routledge.

Ugas, L. (2008). La gestión de los proyectos en las empresas del sector energético. Caso: enelven - CARbozulia. Télématique Revista Electrónica de Estudios Telemáticos, Vol. 7, $\mathrm{N}^{\circ}$ 2, pág. 70-97. República Bolivariana de Venezuela.

Wallace, W. (2014). Gestión de proyectos. 4ta. edición. Edimburgo, Reino Unido: Edinburgh Business School.

Wilson, R. (2015). Mastering project management strategy and processes. Proven methods to meet organizational goals. Estados Unidos de América: Pearson Education.

Zandhuis, A., Snijders, P. y Wuttke, T. (2014). El compañero de bolsillo de la Guía del рмвок. Primera edición. Reino de España: Van Haren Publishing. 